\title{
Professionals' experiences of imaging in the radiography process - A phenomenological approach
}

\author{
Lise-Lott Lundvall, Madeleine Abrandt Dahlgren and Staffan Wirell
}

\section{Linköping University Post Print}

\section{Tweet}

N.B.: When citing this work, cite the original article.

Original Publication:

Lise-Lott Lundvall, Madeleine Abrandt Dahlgren and Staffan Wirell, Professionals' experiences of imaging in the radiography process - A phenomenological approach, 2014, Radiography, (20), 1, 48-52.

http://dx.doi.org/10.1016/j.radi.2013.10.002

Copyright: WB Saunders

http://www.elsevier.com/

Postprint available at: Linköping University Electronic Press

http://urn.kb.se/resolve?urn=urn:nbn:se:liu:diva-103181 
Professionals`experiences of imaging in the radiography process

\title{
-A phenomenological approach
}

\begin{abstract}
Introduction Previous studies on radiographers` professional work have shown that this practice covers both technology and patient care. How these two competence areas blend together in practice needs to be investigated. The professionals`experiences of their work have not been studied in depth, and there is a need to focus on their experiences of the main features of their practice.

The aim: To explore, from the perspective of the radiographer, the general tasks and responsibilities of their work.
\end{abstract}

Method: Data were generated through a combination of open interviews with radiographers and observations of their work with Computer Tomography (CT) and Magnetic Resonance Imaging (M.R.I). The interviews and observations were analysed using an interpretative phenomenological method.

Result: Radiographers' professional work with diagnostic imaging, in a Swedish context, can be viewed as a problem-solving process involving judgments and responsibility for obtaining images that can be used for diagnosis. The examination process comprises three phases; planning, producing the images, and evaluation. In the first phase the radiographer makes judgments on adapting the method to the individual patient, and the second phase involves responsibilities and practical skills for image production. In the third phase, the quality of the images is judged in relation to the actual patient and the imaging process itself.

Conclusions: Radiographers consider that the main features of their professional work are patient safety aspects and their knowledge and skills regarding how to produce images of optimal quality, in the actual circumstances of each examination. 


\section{Keywords: Radiography process, radiographer, phenomenology, diagnostic radiography}

\section{Introduction}

Radiographers' professional scope and national curricula vary in Europe. In some countries, radiography includes both diagnostic imaging and radiotherapy on bachelor level and opportunities for education in radiography on advanced level (1). Even though the professional scope varies, a general aspect of this practice is its combination of operationally complex technology with human communication and patient care. Radiographers' own professional experience of how these aspects blend together in practice has not been investigated. Increased knowledge of this topic would be useful for professionalization and educational purposes. Previous studies can be categorized into three major areas.

The first area concerns studies about radiographers' professional work and identity. Niemi \& Paasivaara (2) studied this topic using a discourse analysis of articles about radiographers` work, all of which had been published in a professional national journal in Finland. Three different discourses were found: a technical discourse describing the hasty technical development, a safety discourse mainly about radiation protection issues, and a professional discourse about education and professionalization. The professional identity is described as dual in character, being socially- and mechanically-oriented. Radiographers work in a technological context, but their activities are also socially-oriented due to the close connection with patient care (2).

Ahonen (3) conducted a concept analysis of radiography in three different contexts: health care, physics and technology. An interdisciplinary comparison showed that constant change due to technical improvements was a common feature. There were special attributes of health care that were not present in the other two contexts; it was dualistic and situation-related. The dualistic aspect shows radiography as both useful for imaging or therapy but also at the same 
time harmful because of the radiation risk. The situation-related aspect means that when using radiation there are safety aspects to take into account, along with the patients`specific condition and needs (3).

A concept analysis of radiographers ` professional work in Finland showed three main aspects: radiation usage/protection, patient care and service for the health care sector. Radiographers' practical work is described as a process comprising planning, implementation and evaluation stages. Safety aspects and interaction with the patient seem to be important parts of their practice (4). Andersson et al. (5) focused on radiographers' professional work in relation to good nursing care. By using the critical incident method, different tasks were identified and categorized into two competence areas; one directly patient-oriented and the other indirectly patient-oriented. This result shows the complexity of the radiographers' work, and that it involves many diverse actions, but it does not capture how the competencies fit together in a working process (5).

The second area concerns research in Sweden regarding the transformation of the practical work and professional role due to usage of digital image production. The main findings are that radiographers have become more independent of radiologists in their work and have gained the responsibility for assessing image quality. Their practical skills in the image production process have changed from being an expert in individualizing exposure parameters to each patient to instead carrying out image processing and operating computers $(6,7,8)$. A third area of research, mainly originating from the United Kingdom (UK), focuses on the role extension of radiographers to include professional tasks and responsibilities belonging to the medical tradition. Government policies and a shortage of radiologists in the UK have facilitated the development of the professional role for some radiographers, who have moved on to an advanced or consulting level. These areas are available for a minority of radiographers; the majority still work with imaging or radiotherapy $(9,10,11,12,13,14)$. 
Radiographers' basic role in diagnostic imaging was investigated in 1999 in the UK. The results described competences covering two areas, patient care and production of high quality diagnostic images $(16,17)$.

Previous research shows that radiographers` professional knowledge, skills and responsibilities cover two main areas: technology and patient care. The rapid technological development in this area has led to a constant change in the practical skills required.

Our research questions were designed to find out radiographers' main responsibilities in their professional work, to identify their general tasks in different imaging technique modalities, and to discover how radiographers establish a balance between patient care and imageproduction.

Research has shown that radiographers' knowledge and skills are expanding into medical and technological fields. For the majority of radiographers the basic role of their profession remains unchanged. This role needs to be understood and articulated.

This paper focuses on the professional practice and knowledge of radiographers in Sweden, where the training in radiography has changed several times. For a time it was linked to nursing education, but in 2000 it became autonomous at bachelor level (15). The professional scope in Sweden is focused on diagnostic and interventional radiography.

\section{The aim}

To explore, from the perspective of the radiographer, the general tasks and responsibilities of their work.

\section{Method}

This qualitative study has used an interpretative phenomenological approach influenced by 
Colaizzi $(18,19,20)$. Methods for data collection in phenomenological studies can be essays, open interviews and observations. Interviews and essays give data about people's articulated lived experiences. Some experiences, things that people do every day, are habituated and therefore difficult to verbalize. For this study, a combination of interviews and observations was therefore used (19). Bracketing was used by the first author because of her own experiences in this practice. The pre-conceived experiences were written down before the study started. The other authors have had no personal experience of this practice.

\section{Sample and data collection}

The first author (L.L) contacted an experienced supervisor at a university clinic and asked if she knew of any radiographers who were experienced and knowledgeable in the field and who would be suitable for interviewing in a study of professional experience. The supervisor nominated five persons who were asked via email about their willingness to take part in the study. Four agreed to participate. Two of these interviewees were asked via email, after the interview, if they knew of any other radiographers who might be suitable for interviewing. Six radiographers were nominated and four of them agreed to participate. Prior to the interview, seven of the interviewees received an interview guide by email, comprising four open-ended questions. The questions were: 1) Describe an ordinary situation that you have experienced when your knowledge as a radiographer was important. 2) Describe a situation when you learned new technology. 3) Describe an ordinary situation when your professional knowledge was important for taking care of patients. 4) What do you know, as a radiographer that other professionals at your place of work (both in your ward but also other professionals you meet during work) do not know? The first interviewee did not receive the interview guide in advance. That was the shortest interview (25 minutes). 
Table1. Demographic data of the interviewees.

\begin{tabular}{|l|l|l|}
\hline Sex & Age (Year) & Years of working experiences \\
& Md (Range) & Md (Range) \\
\hline $3 / 5$ & $39(26-53)$ & $13,5(2-29)$ \\
& & \\
\hline
\end{tabular}

All interviewees worked full-time as radiographers, and four interviewees spent more than half their working time in a specialized field in medical imaging, such as Magnetic Resonance Imaging (MRI), Computer Tomography (CT), and skeletal and intervention radiology.

The interviews varied between 25 minutes and 71 minutes, with a total length of 375 minutes. The interviews were recorded using a voice recorder, and were transcribed verbatim into Swedish by the interviewer.

After a preliminary analysis of the interviews, data collection continued with observations to obtain additional data about general tasks and responsibilities in between different imaging technique modalities, such as CT and MRI. The observations were conducted at a middlesized radiology department in southern Sweden. None of the interviewees worked in that department. The observations were open and were carried out over one week. Each occasion varied in length between two and four hours. There were ten observations in total. Field notes were made directly after each observation. These generated 13 pages of transcripts. The field notes were structured according to three steps: 1) description of the observation, 2) theoretical memos, and 3) reflections on the focus of the next observation.

\section{Data analysis}

The data for analysis consisted of the transcripts from the eight interviews and the field notes from the observations. Each interview and set of field notes was re-read several times. Data 
concerning the "lived experience" of each interviewee's work were underlined in the interviews $(19,20)$. These meaning units were transcribed into a more abstract form in two steps. First, the meaning units were rewritten into the third person and then condensed into a shorter and more abstract form. After analysing the content of the meaning units, they were grouped into themes. Each interview was analysed separately into themes, with a description of each theme derived from the meaning units (19). The themes from all interviews were combined in one general description. The theoretical memos from the observations showed general tasks and responsibilities. These were combined with the general description from the interview data. This made it possible to articulate the radiography process of imaging. The data were analysed in Swedish and reported in English.

The study was approved by the research ethics committee in the medical faculty in Linkoping University (Dnr 2010/74-31). This is the usual way for attaining ethical approval in a medical faculty in Sweden.

\section{Result}

Radiographers`professional work and responsibilities in image-production can be described as a process involving a chain of judgments for attaining a result, namely images that can be used for making a diagnosis. In the following, it will be shown that there are different phases in this process, and we illustrate how radiographers adapt the technology to suit the individual patient.

\section{Planning the examination.}

This phase comprises 1) critically reading and assessing the referral, 2) observing and interviewing the patient to collect missing data, 3) judging communicative capacity, and 4) identifying safety aspects. 
The radiographers emphasized the importance of critical reading and assessing the information in the referral before meeting the patient. During work with CT or MRI, the radiologist chooses the examination method in advance, and when working with conventional methods the radiographers decide which method is most appropriate in relation to the description of the patient`s medical problem and the issue in question.

"From the referral you read which of the patient's problems the remittent is really interested in, what they are asking about. Yes, most of the time they write quite a clear question, saying exactly what they are interested in, but it's not always like that" Interviewee 1

By observing and asking about symptoms, information will be gathered about the patient's medical status. If it differs from the description in the referral, the radiologists judge if the issue in question is appropriate. Observing the patient`s body movements is important, e.g. can pain or functional impairment affect the possibility of attaining visualization of the intended anatomy and pathology. Some of the radiographers said that they can often predict the diagnosis just by looking at the patient, and can thus judge how they should conduct the examination to attain an optimal result.

"In some way, inside your mind, you create an image about how the patient looks inside the body" Interviewee 5

The psychological condition of the patient is also taken into account. Many patients have recently undergone a trauma or have a serious disease. Some have anxiety about undergoing the examination or are worried about the diagnosis. The radiographers emphasized that judging this is important, both from the viewpoint of the patient's wellbeing during the examination but also because it can affect the image quality. Giving these patients some extra time to ask questions or just to talk inspires confidence and cooperation during the 
examination. These patients, if not recognized, may have difficulties in clearly understanding how to co-operate during the examination. An established rapport between the radiographer and the patient eases the co-operation and can prevent motion artefacts.

\footnotetext{
"When you are dealing with an anxious patient you must be able to explain on their level about their situation and what we must do to get a good image to find out what the problem is" Interviewee 4
}

"Before the shoulder examination started, both radiographers were inside and talked to the patient, and another coil was used instead. The patient found it a little bit difficult to lie still and had pain in the shoulder. The radiographers communicated gently, clearly and used body communication for calming, for example they gave the patient a gentle pat before they went out of the room again" Field notes 2010-11-01

Judging the patient`s communicative capacity is important. Low communication capacity due to language difficulties or functional impairment, such as hearing loss, is experienced as a challenge for radiographers to manage because there is limited time for the examination. If not taken into account it can result in motion artefacts.

Finally, the pre-investigation phase concludes with a judgment of the safety aspects of the chosen method in relation to the specific patient. These safety aspects comprise checking the level of radiation for children and fertile women, and ruling out counter indications such as contrast allergy or implants in the body, the latter being a particular safety risk for M.R.I.

\section{Producing the images.}

In this phase, the activities comprise 1) deciding on which protocol to use on the modality, 2) positioning the patient correctly in the machine, 3 ) verification with the patient so that co- 
operation is established, and 4) producing the images.

The radiographers choose the appropriate apparatus protocol in relation to the selected method, the specific patient's condition, capacity, needs, and security risks identified. They explain that their skill lies in their ability to choose the appropriate protocol, and it covers knowledge about the technology beyond button pressing, and gives them the ability to modify the parameters of the modalities when needed. Their responsibility also covers medical technological preparations, such as placing an intravenous catheter in a blood vessel for intravenous contrast or giving oral contrast to the patient.

Before the image production, the radiographers check that the patient is positioned correctly, safely and comfortably. This is to prevent movement during image production, but also to ensure that the correct anatomical area is covered in the images. Sometimes they have to explain to the patients that even though the position is uncomfortable it is important to maintain it, otherwise the image quality may be inadequate.

The radiographers are responsible for communication with the patient during image production. They give the patient advance information about special breathing procedures during image production, and body sensations that may occur when using intravenous contrast.

The practical tasks during image production vary between conventional methods, CT and MRI. A general aspect of this practice is visualization of the same anatomy and pathology, even though it is done with different imaging techniques. During work with conventional imaging techniques, there are practical psycho-motor skills that the radiographer has to master. For example, how to practically position the patient and the radiation tube correctly to attain an optimal image in relation to the specific circumstances for each patient. 
that experienced radiographers have that they can almost see how you must

position the equipment to attain an excellent image" Interviewee 3

During work with CT and MRI it is also important to position the patient accurately but usually the same position is used during image production. Compared to the conventional technique, the radiographer works more with the machine by choosing the right protocols and parameters, timing the contrast injections, and instructing the patient in the best breathing procedures to visualize the appropriate anatomy and pathology.

The radiographers consider that image production is their autonomous professional field because they have practical knowledge and skills about how to handle the equipment and how to take care of the patients during the process.

\section{Evaluating the examination}

After image production, the radiographers evaluate the quality of the images, judging if the correct anatomical area has been covered and whether the quality of the images is sufficient for visualization of the pathology. They believe that they can identify pathology but only do so in situations where it can facilitate the diagnostic process for the patient. An experienced radiographer knows when the image quality is sufficient in relation to the conditions during each examination.

"You know when you have images that are good enough to give an answer with no doubt” Interviewee 6

They write comments to the radiologist if the quality of the images is poor, and explain the conditions. They summarize and conclude the event with the patient and explain the coming phases of the diagnostic process. 


\section{Discussion}

The aim of this study was to explore, from the perspective of the radiographer, the general tasks and responsibilities of their work.

Using phenomenology was useful for acquiring information on the lived experiences of this practice. Analysing in Swedish and reporting in English might be a weakness of this study. Member checking was not used. It could have been an alternative way to strengthen the trustworthiness.

The main findings were: responsibilities in the radiography process cover both safety aspects for the patient, and issues related to how to obtain optimal image quality, taking account of each patient`s needs and ability to cooperate. The safety aspects are related to the use of technology and medicine distribution, in particular the use of radiation and contrast media.

Obtaining optimal image quality depends on how well the radiographer understands the reason for the imaging request, establishes a rapport with the patient, and practically performs the image production. The results shows that the production phase is experienced as an autonomous professional area, and this must be considered in relation to the professional scope of Sweden.

The production phase is described as including actions that have been interpreted as having medical, technological and caring aspects. This result shows that judgements and actions of a medical character start in the planning phase by clarifying the question at issue and taking account of medical safety aspects, for example contrast allergy, in the planning before image production. Positioning the patient correctly for visualization of anatomy is put forward as an 
important task. The radiographers` ability to visualise the images in advance facilitates their decision about how to place the patient safely and correctly before the image production, and is one of the main features of their professional skill. Kelly et al. (9) have described medical aspects of the role of consultant breast radiographer. Former studies about the basic role of diagnostic imaging $(2,16,17)$ have identified the technological and caring aspects but have not specifically identified the medical aspect.

Establishing co-operation with the patient before the examination is important both for attaining high image quality but also for the patient's well-being during the examination. How radiographers do this is briefly described but not really clarified in these results.

In this study, image quality is described as an important professional responsibility. Patients' anxiety is described as a factor that can affect the quality of the images. Possible reasons for anxiety have been presented and some examples of how radiographers cope with this professional dilemma are presented. Törnqvist et al (21) showed that giving detailed information before MRI significantly decreased the presence of motion artefacts on the images but it did not affect patients' level of anxiety before, during, or after the examination. In the evaluation phase, the radiographers say that the image quality is judged in relation to what happened during the examination. The reason is that they understand how various factors can affect the conditions and possibilities of attaining adequate image quality. They know when the quality is enough for diagnosis; 'not perfect but enough'.

The role of radiographers has expanded to include even preliminary diagnostic image interpretation. Thus, radiographers use their knowledge and skills in, for example, the red dotting system, which facilitates the diagnosis process in emergency care (22).

This study shows the radiography imaging process from the professionals` perspective. 
The study contributes a different perspective compared to radiographers' work as described by Ahonen (4). In the latter study, there are some similarities to the results of this study, but Ahonen gives a description on an abstract level.

Taking the professionals' experiences into account is what we would like to emphasise in this study, since there are few studies which have this perspective. On the other hand, the focus on one perspective may also be seen as a weakness. Professional practices are complex arrangements, and to attain deeper knowledge, multiple perspectives must be used. More knowledge is needed, for example about how technology affects this practice. However, how to manage patients`anxiety and create confidence in a short patient meeting also needs to be studied in depth. Moreover, we have not found studies about how radiographers' practice connects or interferes with other practices.

This study was conducted in a Swedish context, with a slightly different professional scope compared to most other European countries. What the radiographers emphasise as important in their practice might be a little different but the combination of technology and patient care in practice and the specific professional dilemmas must have similarities in other countries. Conflicts of Interest: There are no conflicts of interest for this study. 


\section{References}

1. Tuning Template for Radiography in Europe.

http://www.docstoc.com/docs/2633819/Tuning-Template-for-Radiography-in-Europe Available 2013-08-01

2. Niemi A, Paasivaara L. Meaning content of radiographers` professional identity as illustrated in a professional journal-A discourse analytical approach. Radiography 2007; 13(4):258-264.

3. Ahonen S.-M. Radiography- A conceptual approach. Radiography 2008;14(4):288293.

4. Ahonen S.-M. Radiographers` work in Finland-A conceptual review.

European Journal of Radiography 2009; 1(2):61-65.

5. Andersson BT, Fridlund B, Elgán C, Axelsson Å B. Radiographers`areas of professional competence related to good nursing care. Scand J of Caring Science 2008; 22(3):401-409.

6. Larsson W, Aspelin P, Bergquist M, Hillergard K, Jacobsson B, Lindskold L et al. The effects of PACS on radiographer`s work practice. Radiography 2007; 13(3):235240.

7. Larsson W, Lundberg K, Hillergård K. Use your good judgement-Radiographers' knowledge in image production work. Radiology 2009; 15(3):11-e21.

8. Fridell K, Aspelin P, Edgren L, Lindsköld L, Lundberg N. PACS influence the radiographer`s work. Radiography 2009; 15(2):121-33

9. Kelly J, Piper, K, Nightingale, J. Factors influencing the development and implementation of advanced and consultant radiographer practice $-\mathrm{A}$ review of the literature. Radiography 2008;14, Supplement1: e71-e78.

10. Woodford, A. An investigation of the impact/potential impact of a four-tier profession on the practice of radiography - A literature review. Radiography 2006;12(4):318-326. 
11. Ford $\mathrm{P}$. The role of the consultant radiographer - Experience of appointees. Radiography 2010;16(3):189-197.

12. Reeves PJ. Research in medical imaging and the role of the consultant radiographer: A discussion. Radiography 2008;14, Supplement1:e61-e64.

13. Kelly J, Hogg P, Henwood, S. The role of a consultant breast radiographer: A description and a reflection. Radiography 2008; 14:Supplement 1: e2-e10.

14. Hardy M, Legg J, Smith T, Ween B, Williams I, Motto J. The concept of advanced radiographic practice: An international perspective. Radiography 2008; 14, Supplement 1: e15-19.

15. Högskoleförordningen 1993:100. Higher education ordinance in Sweden 1993:100.

16. Williams PL, Berry JE. What is competence? A new model for diagnostic radiographers: Part 1. Radiography 1999;5(4) 221-235.

17. Williams PL, Berry, JE. What is competence? A new model for diagnostic radiographers: Part 2. Radiography 2000;6(1)35-42.

18. Sokolowski R. Introduction to Phenomenology. Cambridge university press; 2000.

19. Colaizzi PF. Psychological research as the phenomenologist views it. In: Valle R, King M, editors. Existential-phenomenological alternatives for psychology. New York: Oxford University Press; 1978, p. 48-71.

20. Szklarski A. Empirical phenomenology. A presentation of the research approach and experiences of one phenomenological study. Nordisk Psykologi 2004;56(4):274-288.

21. Törnqvist E, Mnsson Å, Larsson E-M, Hallström I. Impact of extended written information on patient anxiety and image motion artifacts during Magnetic Resonance Imaging. Acta Radiologica 2006;47(5):474-480.

22. Hardy M, Culpan, G. Accident and emergency radiography: A comparison of radiographer commenting and "red dotting". Radiography 2007; 13(1), 65-71. 
